\title{
The Mixing Of Interplanetary Magnetic Field Lines: A Significant Transport Effect In Studies Of The Energy Spectra Of Impulsive Flares
}

\author{
J. E. Mazur ${ }^{1}$, G. M. Mason²,3 J. R. Dwyer², J. Giacalone ${ }^{4}$, J. R. Jokipii ${ }^{4}$, and E. C. \\ Stone \\ ${ }^{I}$ The Aerospace Corporation, 2350 E. El Segundo Blvd., El Segundo, CA 90245-4691 USA \\ ${ }^{2}$ Department of Physics, University of Maryland, College Park, MD 20742 USA \\ ${ }^{3}$ Institute for Physical Science \& Technology, University of Maryland, College Park, MD 20742 USA \\ ${ }^{4}$ Lunar and Planetary Laboratory, University of Arizona, Tucson, AZ 85721 \\ ${ }^{5}$ California Institute of Technology, Pasadena, CA 91125
}

\begin{abstract}
Using instrumentation on board the $A C E$ spacecraft we describe short-time scale ( $\sim 3$ hour) variations observed in the arrival profiles of $\sim 20 \mathrm{keV}$ nucleon ${ }^{-1}$ to $\sim 2 \mathrm{MeV}$ nucleon $^{-1}$ ions from impulsive solar flares. These variations occurred simultaneously across all energies and were generally not in coincidence with any local magnetic field or plasma signature. These features appear to be caused by the convection of magnetic flux tubes past the observer that are alternately filled and devoid of flare ions even though they had a common flare source at the Sun. In these particle events we therefore have a means to observe and measure the mixing of the interplanetary magnetic field due to random walk. In a survey of 25 impulsive flares observed at ACE between 1997 November and 1999 July these features had an average time scale of 3.2 hours, corresponding to a length of $\sim 0.03 \mathrm{AU}$. The changing magnetic connection to the flare site sometimes lead to an incomplete observation of a flare at $1 \mathrm{AU}$; thus the field-line mixing is an important effect in studies of impulsive flare energy spectra.
\end{abstract}

\section{INTRODUCTION}

Interplanetary particle events from impulsive solar flares have several characteristics that distinguish them from more intense particle events associated with traveling interplanetary shocks. One key characteristic is the pattern of abundance enhancements in the flare events compared to the solar wind composition: ${ }^{3} \mathrm{He}$ is $\sim 10-1000$ more abundant, and $\mathrm{Ne}-\mathrm{Si}$ and $\mathrm{Fe}$ are enhanced by factors of $\sim 3$ to 5 and $\sim 10$, respectively (e.g. Reames [1] and references therein). An observer at $1 \mathrm{AU}$ detects the electrons and ions from flares that are only at western solar longitudes near the intersection of the nominal interplanetary magnetic field line and the solar corona (e.g. Kahler et al. [2]; Cane, McGuire, \& von Rosenvinge [3]). The unique abundance signatures that possibly result from wave-particle resonances (e.g. Temerin \& Roth [4]; Miller \& Viñas [5]), the high ionization states that imply temperatures above $10 \mathrm{MK}$ (Luhn et al. [6]), and the requirement of favorable magnetic connection to a flare site all indicate that the energetic particle source is close to the Sun, within a solar radius of the photosphere.

The propagation of flare electrons and ions from the flare site to $1 \mathrm{AU}$ yields another characteristic of these particle events. The 
acceleration process may take only tens of seconds to fully accelerate ions and electrons (Miller \& Viñas [5]). This is much shorter than the minutes, hours, or even nearly day-long time scales associated with the particle propagation along magnetic field lines to 1 AU. The distribution of particle speeds therefore produces a measurable velocity dispersion wherein propagation effects dominate. Larson et al. [7] found that the effect of velocity dispersion revealed details of the propagation of $\sim 0.14$ to $100 \mathrm{keV}$ electrons from impulsive flares within a magnetic cloud. They interpreted the numerous abrupt decreases of the electron fluxes as signatures of the disconnection of one end of the cloud's magnetic field from the solar corona. The electron event profiles in the study of Larson et al. [7] lasted as long as $\sim 6$ hours.

Here we show observations of the temporal structures in the arrival profiles of $\sim 20 \mathrm{keV}$ nucleon ${ }^{-1}$ to $5 \mathrm{MeV}$ nucleon ${ }^{-1}$ impulsive solar flare ions made between 1997 November and 1999 July with instrumentation on board the $A C E$ spacecraft (Mazur et al. [8]). At the lowest energies the ions have minimum times-of-flight from the flare to $1 \mathrm{AU}$ of $\sim 24$ hours, with some variation due to the solar wind speed and the length of the resulting magnetic field line. These low energy ions propagate to $1 \mathrm{AU}$ faster than the solar wind but slower than the energetic electrons that Larson et al. [7] discussed. We therefore use these ions as probes of pre-existing interplanetary magnetic field structures on the scale of $\sim 0.03 \mathrm{AU}$, allowing us to study the magnetic connection to a flare with ions over a much longer time scale than previously possible.

\section{OBSERVATIONS}

The ion observations presented here were made with the Ultra-Low Energy Isotope Spectrometer (ULEIS) sensor on board the Advanced Composition Explorer $(A C E)$ spacecraft, which was launched into orbit about the L1 Lagrangian point in late 1997 (Stone et al. [9]). ULEIS is a time-of-flight mass spectrometer that measures composition and energy spectra of $\mathrm{H}-\mathrm{Ni}$ in the energy range of $\sim 0.02-$ $10 \mathrm{MeV}$ nucleon $^{-1}$ (Mason et al. [10]). We have observed numerous interplanetary particle events whose abundances and occasional associations with Type-III radio bursts and streaming $\sim 100 \mathrm{keV}$ electrons indicate that the ions were accelerated at an impulsive flare site (e.g. Mason et al. [11]; Reames [1]).

\section{Event Profiles: Impulsive Versus Shock- Associated Energetic Particles}

Figure la-d shows an example of impulsive flares observed with $A C E$ during 1999 January 9-10. Figure 1a shows the energy ( $\mathrm{MeV}$ nucleon $\left.{ }^{-1}\right)$ of $\mathrm{H}-\mathrm{Fe}$ ions as a function of their arrival time. The histogram in Figure $1 \mathrm{~b}$ plots the smoothed ion count rate binned in 14-minute intervals in order to better show the intensity variations. At least 2 particle injections occurred within this 2-day interval: one event began at $\sim 1400 \mathrm{UT}$ on 9 January 1999, and particles at $1 \mathrm{MeV}$ nucleon $^{-1}$ from another injection arrived at $1 \mathrm{AU}$ on 1999 January 10 at $\sim 0900$ UT

We focus on the more intense event beginning on 1999 January 9 at 1300UT. The velocity dispersion tells us that the ions in this event are from the same source at the Sun; we could not conclude this from the event counting rates alone shown in Figure 1b. Notice the occasional interruptions of the event profile on the time scale of $\sim 1$ hour wherein the particle counting rate decreased by a factor of 5-10 (e.g. at 1920 UT on 1999 January 9). The modulation of the event profile occurred simultaneously across all energies, and did not correlate with large changes in the direction of the local interplanetary magnetic field (Figure $1 \mathrm{c}-\mathrm{d}$ ). The transitions from the relatively high intensity event rates to pre-existing particle rates were quite sharp, lasting $<2.5$ minutes.

In order to quantify these short-term intensity variations, we analyzed the event histogram of Figure $1 \mathrm{~b}$ in the following way: beginning with the first arrival of ions from the flare at 1407UT on 1999 January 9 , we used the velocity dispersion profile in Figure la to distinguish the particles of interest from the low fluxes of particles from previous events. From this time, we marked the end of a sub-interval (labeled 1 in Figure 1b) and the beginning of another (labeled 2) when the counting rate increased by a factor of $\sim 1.3$. This procedure was continued through the entire event, measuring the start and end times with a peak-to-valley ratio criterion of $\sim 1.3$. This particular threshold for deciding when to label a new sub-interval was sufficiently low to pick out the features clearly seen with the eye in the velocity profiles. The velocity dispersion profile clearly shows when the next event began on 1999 January 10 at 0910UT, where we measured a new set of intervals using the same technique. We return to the statistics of the sub-intervals measured in these and other events in the next section. 


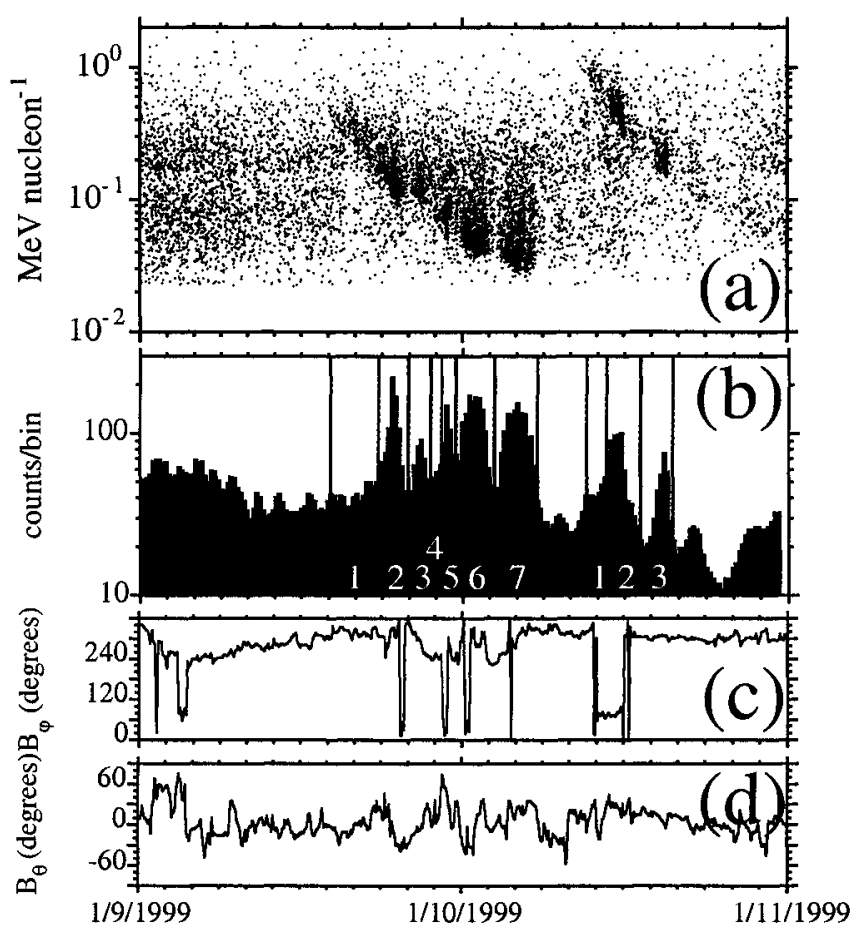

FIGURE 1. Energetic particle velocity dispersion and interplanetary magnetic field direction during 1999 January 9-10. (a) H-Fe ion energy versus time; (b) histogram of ion count rate; (c) magnetic field azimuth direction in the geocentric-solar-ecliptic (GSE frame); (d) magnetic field altitude direction (GSE frame).

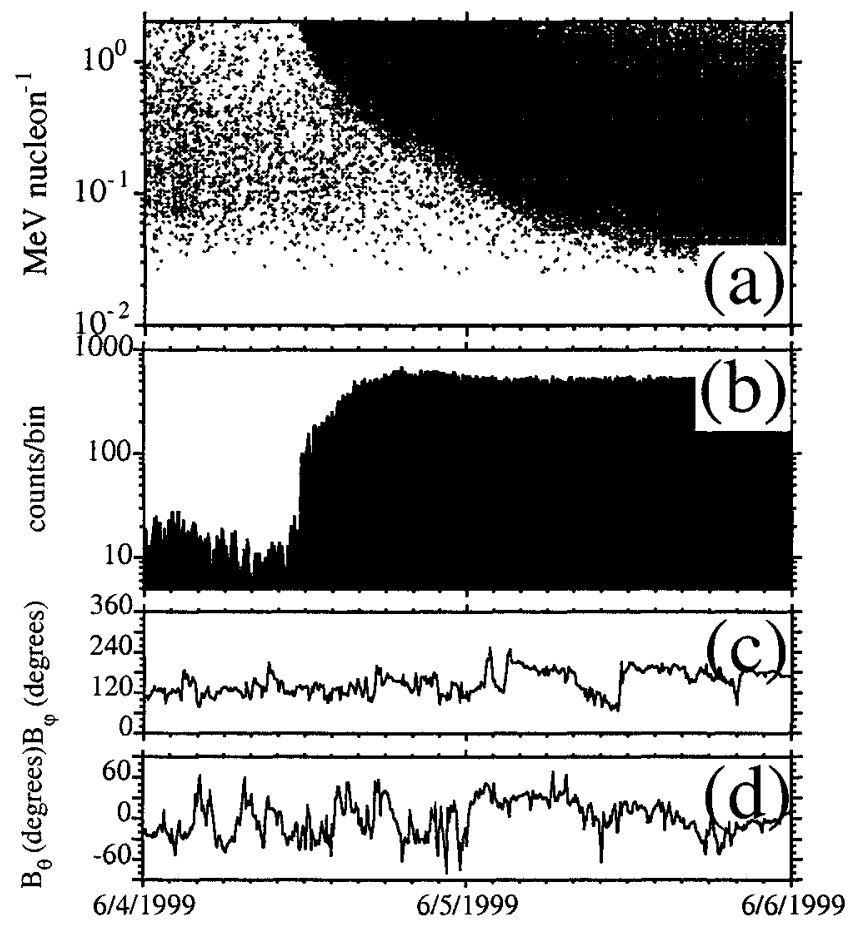

FIGURE 2, Oxygen velocity dispersion and interplanetary magnetic field direction during the 1999 June 4 event. 
We next contrast the impulsive time profile of Figure 1 with an event on 4 June 1999 (Figure 2a-d) that was associated with a coronal mass ejection and interplanetary shock. This event also had velocity dispersion for ions and electrons with an onset near 0700UT on 1999 June 4, but we did not observe intensity variations of the kind that were seen in the impulsive flare of 1999 January 9 . We also note that the interplanetary magnetic field direction changed by 10 's of degrees without any significant effect on the event's profile (Figures $2 c \& d$ ).

\section{Size Of The Fine Structure}

Figure 1 showed an example of an impulsive solar flare event that had significant structure in its time-of-arrival profile. In order to characterize these structures more fully and to determine how often they occur, we surveyed the ULEIS observations from 1997 November to 1999 July for events that had the following characteristics: (1) clear velocity dispersion in heavy ions $(Z>2)$, similar to that shown in Figure 1 ; (2) $\mathrm{Fe} / \mathrm{O} \sim 1$ as is characteristic of particles from impulsive flares; (3) low-intensity events, similar to Figure 1, in order to eliminate the heavy-ion rich onsets commonly seen in shock-associated particle events. We found 25 impulsive flare particle events from 1997 November to 1999 July that satisfied the survey criteria and list them in Table 1.

In order to characterize the time scale of the flare particle intensity variations, we applied the same technique as presented in Figure 1 above (peakto-valley ratio of $\sim 1.3$ ) to each event of Table 1 . To show the wide variety of impulsive event profiles at 1 $\mathrm{AU}$, we plot in Figure 3 the energy/nucleon versus time of heavy ions (C-Fe) in four separate impulsive event periods (events \#5, 7-11,12-13, and 21 of Table 1). Each panel covers a 2-day interval; the color scale is logarithmic in counts/sec with an arbitrary normalization. The event of 1998 April 12 had a $\sim 6$ hour-long interruption in its profile, in contrast to the many shorter-lived intervals observed in the 1999 January 9 events. Note that the ions near 1 $\mathrm{MeV} /$ nucleon that arrived late on 11 April 1998 are part of the same event that began again after the drop-out near $\sim 0200$ on 12 April 1998; only the velocity dispersion shows the relation between these two flux increases.

Figure $4 \mathrm{a}$ shows the distribution of the subinterval durations of all 25 events in the survey. The average duration was 3.2 hours, with the bulk of the sub-intervals shorter than $\sim 3$ hours. We next took the average solar wind speed, also measured on $A C E$, within each sub-interval to calculate the spatial size of the region filled with energetic particles that convected past the spacecraft. Note that this estimate of the spatial size does not include any possible effects of the flux tube geometry or its angle with respect to the radial solar wind flow and is therefore a rough estimate. The histogram in Figure $4 \mathrm{~b}$ shows the distribution of resulting sizes, with an average of $4.7 \times 10^{6} \mathrm{~km}$ or $\sim 0.03 \mathrm{AU}$. In the discussion below we address how this estimate compares to previous measurements of the size of structures observed in the solar wind plasma.

\section{Magnetic Connection Effects On Energy Spectra}

The impulsive flares shown in the previous section make the case that the structure of the interplanetary magnetic field has a large effect on the particle time-intensity profiles. The connection effects may also have large impacts on measurements of the particle energy spectra. As records of the energy distribution of the particles that escape from the flare, it is critical to know whether features such as peaked spectra represent a source effect or a transport effect (e.g. Reames, Richardson, \& Wentzel [12]).

As an extreme example how an incompletely observed event distorts the particle energy spectra, in Figure 5 we show the dispersion profile of $\mathrm{Fe}$ ions from an impulsive flare on 16 May 1998 (event \#6). The ions arrived at ACE only within a $\sim 4$-hour long interval, just after a compressed region of solar wind and just before turbulent magnetic fields associated with a highspeed solar wind stream. The curve shows the expected profile of zero-degree pitch angle particles traveling 1.1 AU along an archimedian spiral from a flare that occurred early on 16 May 1998 at W44. We see that the field lines from this flare corotated past ACE just at the right time for ions between $\sim 100$ and a few hundred $\mathrm{keV} /$ nucleon to arrive at $1 \mathrm{AU}$.

The velocity dispersion yields energy spectra that are peaked in energy as show in Figure 6. The "pulse" of particles moved from higher to lower energy during the 4-hour long interval, resulting in spectra that rolled-over arose because of the observer's changing connection. In this example, the velocity dispersion and interplanetary context together show that we observed only a subset of a longer event and that processes near the source did not lead to the peaked energy spectra. 

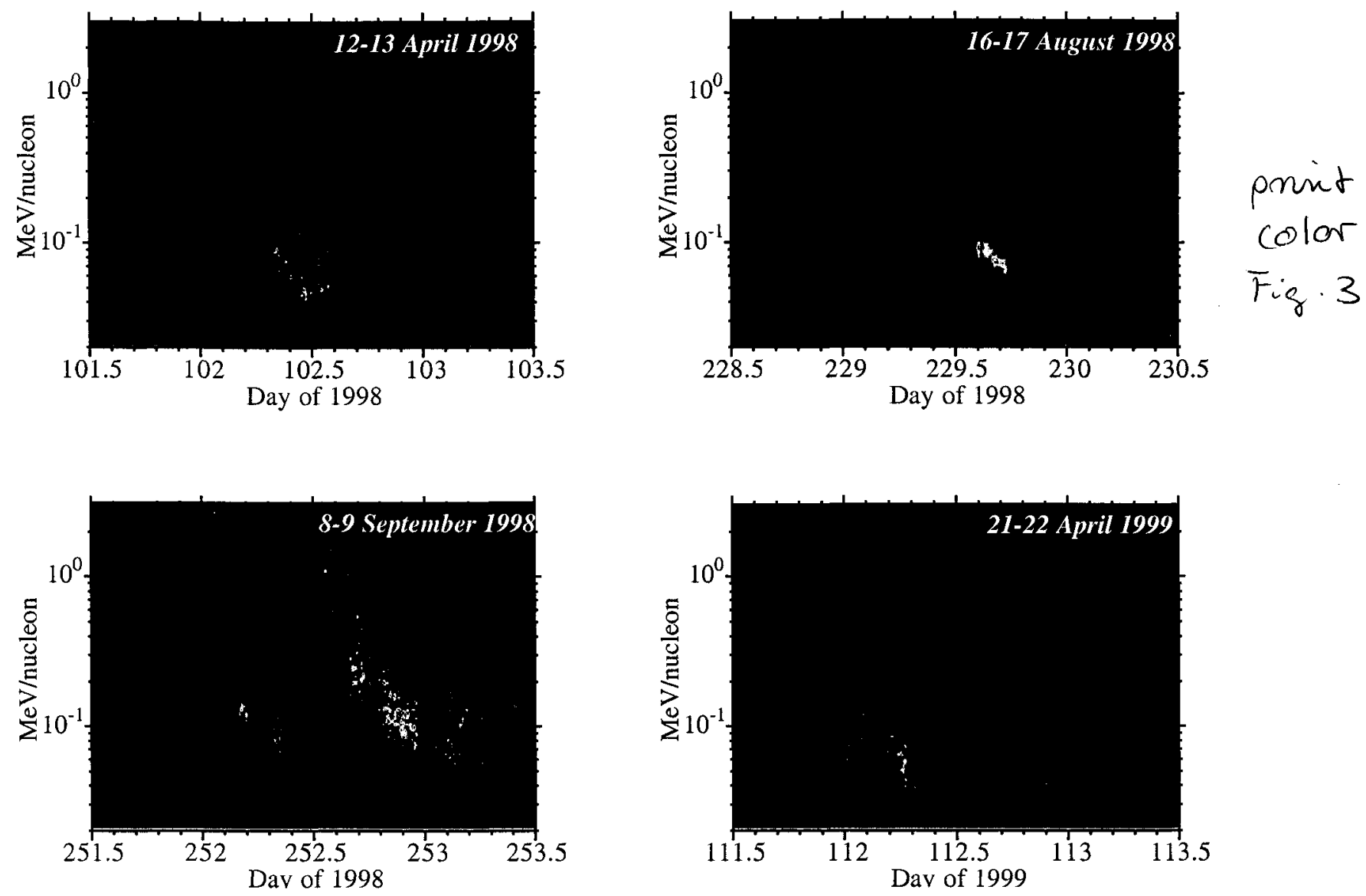

FIGURE 3. Velocity dispersion profiles of heavy ions in several impulsive flare events at $1 \mathrm{AU}$.
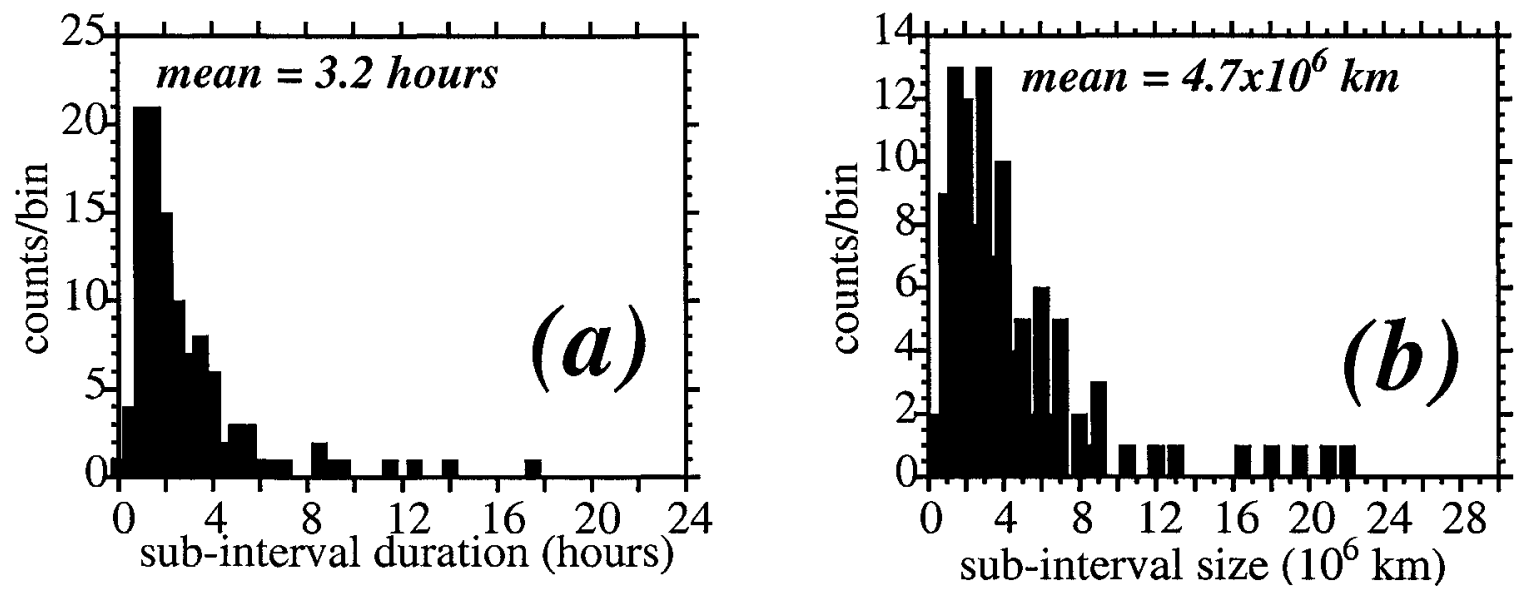

FIGURE 4. Time and size distributions of sub-intervals in 25 impulsive flares. 
TABLE 1. Impulsive event time periods

\begin{tabular}{|c|c|c|c|c|c|}
\hline Event & Start ${ }^{a}$ & Stop & Duration $^{\mathrm{b}}(\mathrm{hr})$ & $\begin{array}{l}\text { Number of } \\
\text { sub-intervals }\end{array}$ & $\begin{array}{l}\text { Average } \\
\text { solar } \\
\text { wind } \\
\text { speed } \\
\left(\mathrm{km} \mathrm{sec}^{-1}\right)\end{array}$ \\
\hline \multicolumn{6}{|c|}{1997} \\
\hline 1 & Nov $14,13: 34$ & Nov $15,12: 58$ & 23.4 & 3 & 342 \\
\hline 2 & Nov $15,4: 19$ & Nov $15,22: 34$ & 18.3 & 4 & 347 \\
\hline 3 & Nov $22,18: 28$ & Nov $23,2: 24$ & 7.93 & 3 & 504 \\
\hline 4 & Nov $22,19: 55$ & Nov $23,2: 25$ & 6.51 & 5 & 514 \\
\hline \multicolumn{6}{|c|}{1998} \\
\hline 5 & Apr 11, 19:18 & Apr $12,19: 35$ & 24.3 & 5 & 382 \\
\hline 6 & May $16,8: 09$ & May $16,11: 52$ & 3.73 & 2 & 507 \\
\hline 7 & Aug $15,12: 53$ & Aug $16,6: 33$ & 17.6 & 7 & 369 \\
\hline 8 & Aug 17, 2:35 & Aug $17,17: 30$ & 14.9 & 2 & 336 \\
\hline 9 & Aug 17, 6:26 & Aug $18,10: 45$ & 28.3 & 11 & 310 \\
\hline 10 & Aug $17,21: 11$ & Aug $18,10: 12$ & 13.0 & 2 & 298 \\
\hline 11 & Aug $18,10: 33$ & Aug $18,23: 31$ & 12.9 & 6 & 330 \\
\hline 12 & Sep 8, 19:58 & Sep $9,12: 17$ & 16.3 & 8 & 359 \\
\hline 13 & Sep 9, 7:37 & Sep 9, 23:00 & 15.4 & 4 & 373 \\
\hline \multicolumn{6}{|c|}{1999} \\
\hline 14 & $\operatorname{Jan} 9,14: 07$ & $\operatorname{Jan} 10,5: 31$ & 15.4 & 7 & 412 \\
\hline 15 & $\operatorname{Jan} 10,9: 10$ & $\operatorname{Jan} 10,15: 31$ & 6.35 & 3 & 411 \\
\hline 16 & Feb $20,7: 25$ & Feb 20, 20:02 & 12.6 & 1 & 433 \\
\hline 17 & $\operatorname{Mar} 21,21: 06$ & $\operatorname{Mar} 22,3: 27$ & 6.35 & 2 & 327 \\
\hline 18 & $\operatorname{Mar} 25,23: 00$ & $\operatorname{Mar} 26,7: 35$ & 8.57 & 3 & 441 \\
\hline 19 & Apr 2, 16:49 & Apr 3, 9:29 & 16.7 & 4 & 420 \\
\hline 20 & Apr 5, 9:31 & Apr 6, 21:23 & 35.9 & 6 & 524 \\
\hline 21 & Apr $21,19: 12$ & Apr $22,14: 00$ & 18.8 & 7 & 467 \\
\hline 22 & May $12,9: 38$ & May $12,17: 15$ & 7.6 & 6 & 425 \\
\hline 23 & Jun $6,2: 32$ & Jun $9,17: 18$ & 14.8 & 6 & 600 \\
\hline 24 & Jul 3, 20:31 & Jul 4, 12:02 & 15.5 & 4 & 456 \\
\hline 25 & Jul 4, 6:12 & Jul 4, 20:26 & 14.2 & 1 & 435 \\
\hline
\end{tabular}

all times UT, 1997-1999.

${ }^{b}$ Duration of heavy ion fluxes, $\sim 0.02-2 \mathrm{MeV}$ nucleon ${ }^{-1}$.

${ }^{c}$ Sub-intervals as defined in section 2.1 .

d 1 -hour averaged solar wind speeds from ACE/SWEPAM. 

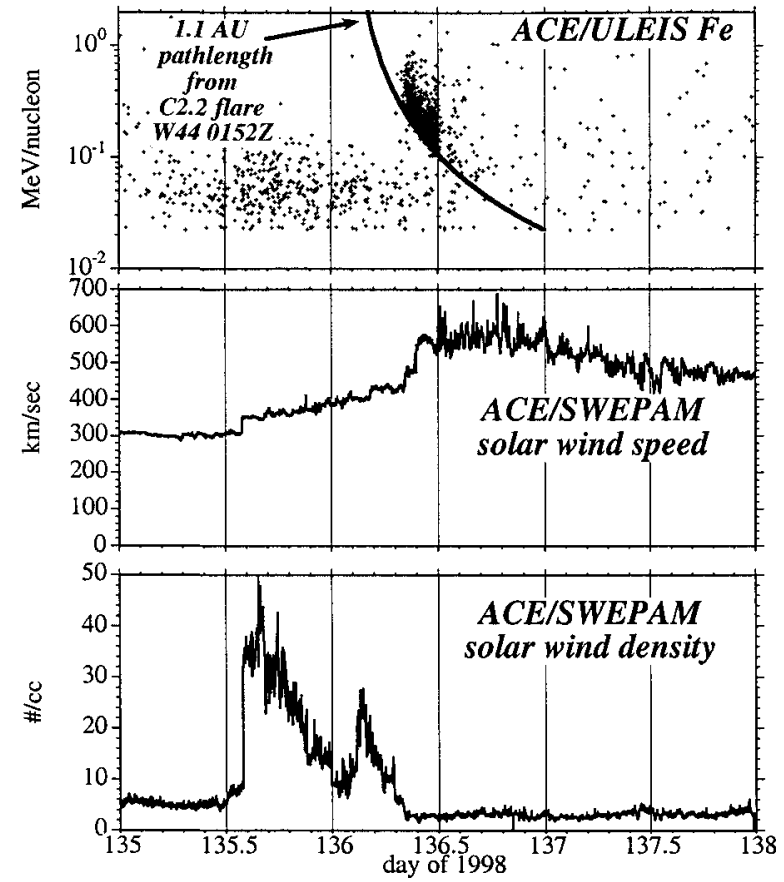

FIGURE 5. Fe dispersion profile and solar wind context for the 16 May 1998 impulsive flare.

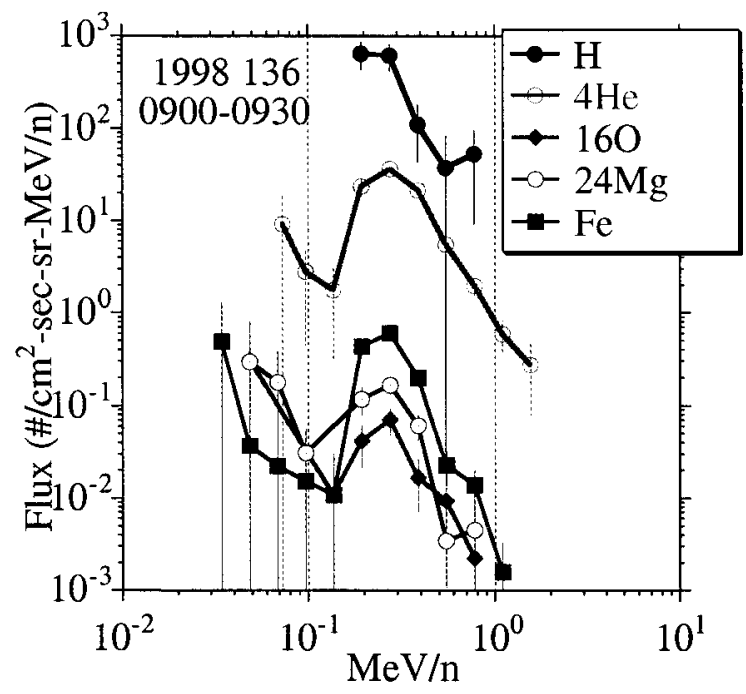

FIGURE 6. Average energy spectra in a 30 -minute period during the 16 May 1998 flare.

\section{DISCUSSION}

Using the $A C E / U L E I S$ instrument we have surveyed energetic particles from 25 impulsive solar flares and have found that the low energy ion intensity profiles exhibited sharp ( $<2.5$ minutes) boundaries and occasional drop outs. The intensity changes occurred simultaneously across all energies and generally were not coincident with signatures of the local interplanetary magnetic field. Since the spacecraft must be magnetically connected to the flare sites in order to see the escaping ions, and since impulsive flare particles originate at compact sites, the observed waxing and waning of the particle intensities is puzzling.

These effects may be caused as follows (Mazur et al. [8]; Giacalone, Jokipii, \& Mazur [13]). The solar wind continuously convects magnetic flux tubes that are connected to a solar active region to 1 $\mathrm{AU}$ and beyond. During the convection to $1 \mathrm{AU}$, which takes about 3-4 days, the footpoints of these flux tubes become mixed with the footpoints of other flux tubes that are not magnetically connected to the active region. The footpoint motion has the effect that adjacent flux tubes at $1 \mathrm{AU}$ can be significantly separated at the Sun, and, conversely, that adjacent flux tubes at the Sun may be separated at $1 \mathrm{AU}$ on scales of $\sim 0.01 \mathrm{AU}$. The flare occurs and populates those flux tubes that are connected to the acceleration site with energetic particles; the other unconnected flux tubes remain empty. The particles within each connected flux tube arrive at $1 \mathrm{AU}$ with velocity dispersion. As the mixed flux tubes move past $A C E$, we see either filled flux tubes and hence the on-going particle velocity dispersion, or empty flux tubes and hence the particle drop-outs. In this picture, the local interplanetary magnetic field does not necessarily correlate with the dramatic intensity changes we have seen. Variations of the particle acceleration process at the Sun are ruled out as a cause of the intensity changes because the drop-outs occur simultaneously at all energies.

The effects of field-line mixing are not as obvious in the more intense particle events associated with coronal mass ejections and interplanetary shocks. We can understand this as a result of the relative sizes of the acceleration sites: in shockrelated events, the temporal structure observed at 1 $\mathrm{AU}$ reflects the ever-changing magnetic connection of an observer to the expanding shock that covers a wide extent in heliolongitude (e.g. Cane, Reames, \& von Rosenvinge [14]). Consequently, any flux tube that $A C E$ intersects will be populated with energetic ions as the shock propagates outward. This is not the case for impulsive flares because the acceleration site of an impulsive flare is much smaller and therefore it is more likely that an observer's magnetic connection will wax and wane depending on the detailed magnetic topology near the flare. 
These effects have only been glimpsed in prior studies of impulsive flare heavy ions (e.g. Reames, Richardson, \& Wenzel [12]) mainly because these earlier studies covered energies above $\sim 1$ $\mathrm{MeV} /$ nucleon. At these higher energies, the particle events do not last as long (c.f. Figure 1a) making it less likely to see structure on the time scale of an hour. The falling energy spectra also make the statistical accuracy poorer at the higher energies, putting the detection of such structures reported here outside the capability of prior studies of impulsive flares.

Anderson and Dougherty [15] observed numerous instances of modulation of electron and ion intensities during long-lasting solar or interplanetary events. Also, Buttighofer [16] noted similar abrupt modulations of low-energy electrons observed on Ulysses that had no obvious coincident plasma signature. We believe that the particle channels observed by Anderson and Dougherty [15] and Buttighofer [16] are the same kind of interplanetary structures we have examined in this work. We also note that the structures discussed here may have led to the electron disconnection events Larson et al. [7] observed within a magnetic cloud.

The interplanetary magnetic field structure that we have deduced from these observations should be related to measurements of the solar wind and the interplanetary magnetic field. Matthaeus et al. [15] studied the characteristic time of correlations between the interplanetary magnetic field and solar wind speed measured at two different times from a single spacecraft. The average correlation time (3.8 hours) and length $\left(4.9 \times 10^{6} \mathrm{~km}\right)$ measured in the solar wind by Matthaeus et al. [17] are indeed similar to the average flare sub-interval duration ( 3.2 hours) and inferred length $\left(4.7 \times 10^{6} \mathrm{~km}\right)$ reported here.

We have seen cases with ACE where the changing magnetic connection to the flare causes an incomplete observation of the particle event. The resulting energy spectra may then have features such as low-energy roll-overs that arise not because of processes such as energy loss, but because of the mixing of the interplanetary magnetic field. Measurements well below $\sim 1 \mathrm{MeV} /$ nucleon where the fluxes are highest are key to observing the intermittent magnetic connection. In future studies of impulsive flare energy spectra we can thus account for a significant transport effect.

Giacalone, Jokipii, \& Mazur [13] \& [18] discuss a model that relates these observations to a random walk of the magnetic field lines that are line- tied in the supergranulation network of the sun's photosphere. Particle events on the scale of a supergranule (tens of thousands of $\mathrm{km}$ ) in the model show structures at $1 \mathrm{AU}$ that are similar to the observations discussed here.

\section{ACKNOWLEDGMENTS}

We thank the many members of the $A C E$ instrument teams who have contributed to the success of the ACE mission, and acknowledge the special efforts of R. E. Gold and S. M. Krimigis. We thank C. Smith and the ACE/MAG team for the ACE magnetic field measurements reported here, and D. McComas, R. Skoug, and the ACE/SWEPAM team for solar wind measurements used in the analysis. Contract number Q295801 between the University of Maryland and The Aerospace Corporation supported this work.

\section{REFERENCES}

1. Reames, D. V., Space Sci. Rev., 90, 413-489 (1999).

2. Kahler, S. W., Lin, R. P., Reames, D. V., Stone, R. G., \& Liggett, M., Solar Phys., 107, 385-394 (1987).

3. Cane, H. V., McGuire, R. E., \& von Rosenvinge, T. T., ApJ, 301, 448-459 (1986).

4. Temerin, M. \& Roth, I., ApJ, 391, L105-L108 (1992).

5. Miller, J. A. \& Viñas, A. F., ApJ, 412, 386-400 (1993).

6. Luhn, A., Klecker, B., Hovestadt, D., \& Möbius, E., ApJ, 317, 951-955 (1987).

7. Larson, D. E. et al., Geophys. Res. Letters, 24, 19111914 (1997).

8. Mazur, J. E., Mason, G. M., Dwyer, J. R., Giacalone, J., Jokipii, J. R., \& Stone, E. C., ApJ. Letters, L79-L83 (2000).

9. Stone, E. C., Frandsen, A., M., Mewaldt, R. A., Christian, E. R., Margolies, D., Ormes, J. F., \& F. Snow, Space Sci. Rev., 86, 1-22 (1998).

10. Mason, G. M. et al., Space Sci. Rev., 86, 409-448 (1998)

11. Mason, G. M., Reames, D. V., Klecker, B., Hovestadt, D., \& von Rosenvinge T. T., ApJ, 303, 849-860 (1986).

12. Reames, D. V., Richardson, I. G., \& Wenzel, K. P., ApJ, 387, 715-721 (1992).

13. Giacalone, J., Jokipii, J. R., \& Mazur, J. E., $A p J$. Letters, $L 75-L 78$, (2000).

14. Cane, H. V., Reames., D. V., \& von Rosenvinge, T. T., J. Geophys. Res., 93, 9555-9567 (1988).

15. Anderson, K. A., \& Dougherty, W. M., Sol. Phys., 103, 165-175 (1986).

16. Buttighoffer, A., $A \& A$., 335, 295-302 (1998).

17. Matthaeus, W. H., Goldstein, M. L., \& King, J. H., J. Geophys. Res., 91, 59-69 (1986).

18. Giacalone, J., Jokipii, J. R., \& Mazur, J. E., "SolarEnergetic Particles vs. Global Cosmic-Ray Diffusion", these proceedings, (2000). 